\title{
ALTERNATIVE APPROACH TO INFINITY
}

\author{
PETER G. BERGMANN
}

Syracuse University, U.S.A.

\begin{abstract}
Following Penrose's construction of space-time infinity by means of a conformal construction, in which null-infinity is a three-dimensional domain, whereas time- and space-infinities are points, Geroch has recently endowed space-infinity with a somewhat richer structure. An approach that might work with a large class of pseudo-Riemannian manifolds is to induce a topology on the set of all geodesics (whether complete or incomplete) by subjecting their Cauchy data to (small) displacements in space-time and Lorentz rotations, and to group the geodesics all of whose neighborhoods intersect into equivalence classes. The quotient space of geodesics over equivalence classes is to represent infinity. In the case of Minkowski, null-infinity has the usual structure, but $I^{0}, I^{+}$, and $I^{-}$each become three-dimensional as well.
\end{abstract}

